\title{
Pediatric bone tumors in Croatia, a single oncology centre experience
}

\section{Introduction}

Malignant bone tumors are recognized as rare diseases following the already estimated incidence which is well known, with osteosarcoma as the most common bone tumor [1, 2]. Median incidence rate for malignant childhood tumors in Croatia is 163 new cases during period 1987-2014, age 0-19 according to Croatian National Cancer Registry (CNCR) data ( $\triangleright$ Fig. 1).

\section{Materials and Methods}

This study consists of incidence rates and long-term survivor rates along with the analysis of risk factors of childhood bone tumors in the period of 1990-2017 for children and young adolescents who were treated in Children's Hospital Zagreb. There were 140 patients with osteosarcoma, with predominant male patients $58,6 \%(n=82)$, median age at diagnosis of 14 years (0-32y) and median follow-up time of 15 years for all patients.

The number of Ewing sarcoma family patients in the same period was 119 , with more male patients $57 \%(n=68)$, median age at the diagnosis was 12,5 years $(0,25-21$ years) with a median follow-up of 15 years.

Patients were treated accordingly due to a protocol study schemes that have been changing during the follow-up period of 28 years.

For treating osteosarcoma Rosen T and COSS multimodal protocol were used for period 1990-2004 $(n=76)$ and from 2005 EURAMOS protocol $(n=64)$.

The Ewing sarcoma family of tumors were treated according to MMT and CWS study protocol schemes, and after 2000 according to EURO EWING 99 and Ewing 2008, which implied the implementation of high dose therapy with transplantation of autologous peripheral hematopoietic stem cells rescue (autoPBSCT).

\section{Results}

Overall 5-year probability survival for osteosarcoma from 1990-2017 was $60 \%$, having in consideration the total number of 140 pa-

\author{
Number of new cases, $0-19$, CNCR
}

250

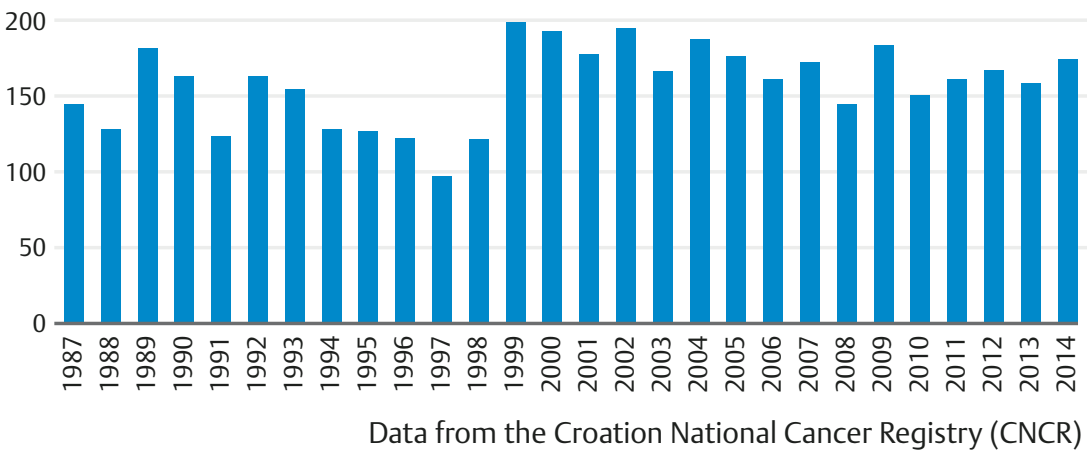

> Fig. 1 Incidence of Childhood Cancer in Croatia from 1987-2014, range: 98-201, median: 163.

- Tab. 1 Characteristics of osteosarcoma patients, 1990-2017.

Demographic and diagnostic data

\section{No of patients $(n=140)$}

No of survivors $(n=78)$

\section{Sex}

Male

Female

82

$51 \%(n=42)$

2. Age at diagnosis

\begin{tabular}{l|l|l|}
\hline $0-5 y$ & 5 & $60 \%(n=3)$ \\
\hline $6-11 y$ & 22 & $59 \%(n=13)$ \\
\hline $12-17 y$ & 96 & $53 \%(n=51)$ \\
\hline$>18$ & 17 & $52,9 \%(n=9)$
\end{tabular}

\section{Disease}

localised (at time of diagnosis)

local. disseminated (at time of diagnosis)

metastatic (during course of disease)

\section{Response to Chemo HUVOS}

poor (grade I+ II)

good (grade III + IV)

*no data

\begin{tabular}{|l|l|}
\hline 70 & $82 \%(n=58)$ \\
\hline 70 & $24 \%(n=17)$ \\
\hline 43 & $9 \%(n=4)$ \\
\hline $\mathbf{n = 1 1 1}$ & $\mathbf{5 4 \% ( n = 6 0 )}$ \\
\hline 65 & $32 \%(n=21)$ \\
\hline 46 & $84 \%(n=39)$ \\
\hline 29 & $(n=14)$ \\
\hline
\end{tabular}

*due to metastatic disease/local invasion -mutilation hazard/primary resection

tients. The survival rate due to sex was $51 \%$ for boys and $58 \%$ for girls, and due to age $60 \%$ for children younger than 5 years, and $59 \%$ for children between 6 and 11 years. $53 \%$ was survival rate for children above 12 years of age. Also, when analysing the stage of disease, the children with localised disease had $82 \%$ survival $(n=58)$, locally disseminated had $24 \%(n=17)$ and the group of patients with metatastatic disease had a $9 \%(n=4)$ survival rate. Survival rate according to chemotherapy response and HUVOS grading was as follows, for good response $84 \%(n=39)$ and for poor response of $32 \%(n=21$; $>$ Table 1$)$. The 5 -years survival probability rate showed for localised disease $86 \%(n=70)$ vs. locally disseminated disease of $34 \%$ ( $\triangleright$ Fig. 2). 


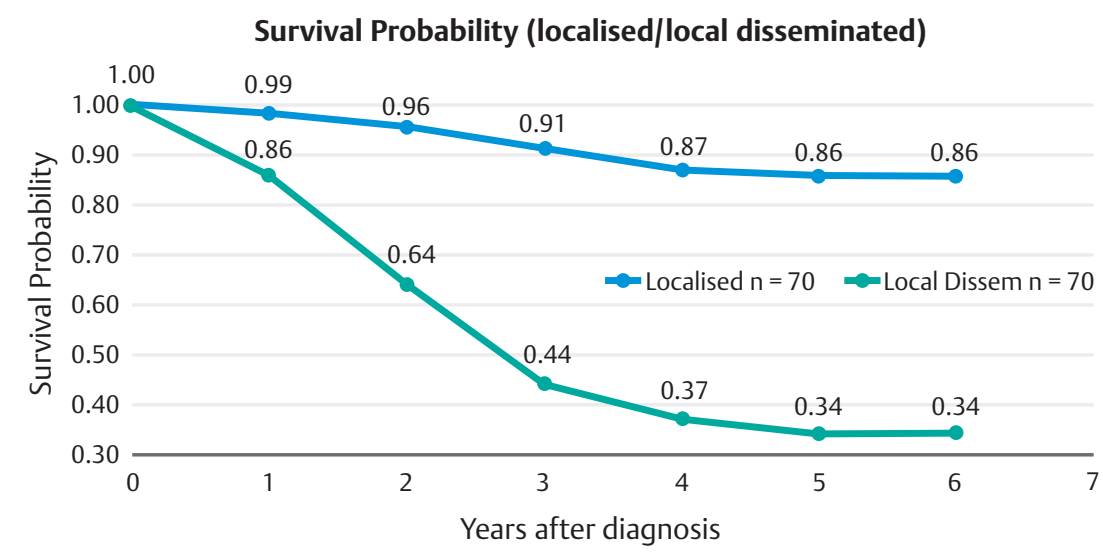

- Fig. 2 5-year survival probability for osteosarcoma patients, localized disease vs. locally disseminated, 1990-2017.

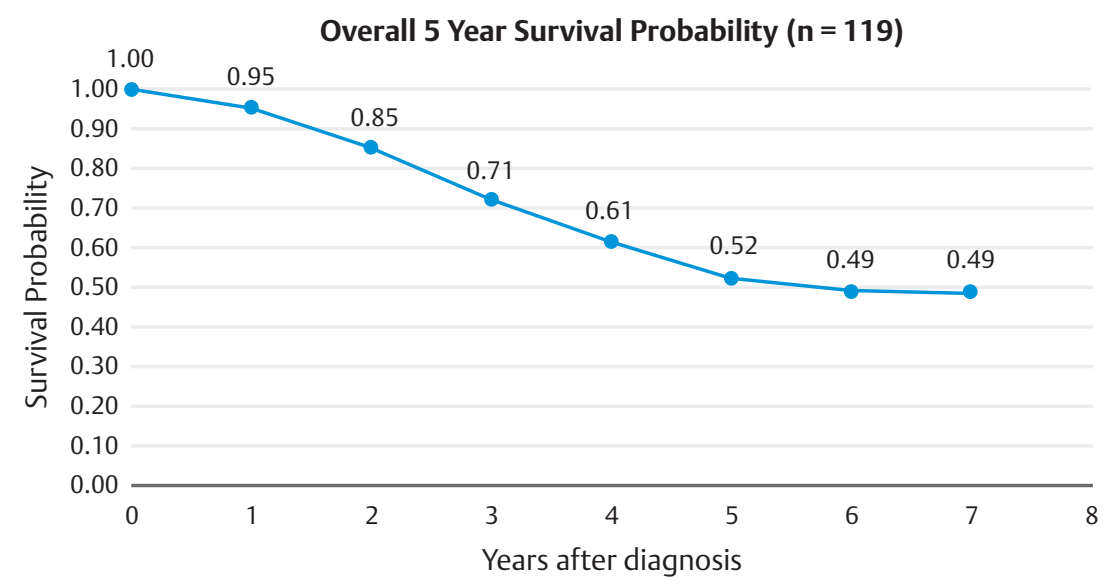

- Fig. 3 5-year probability survival rate for Ewing sarcoma patients ( $n=119), 1990-2017$.

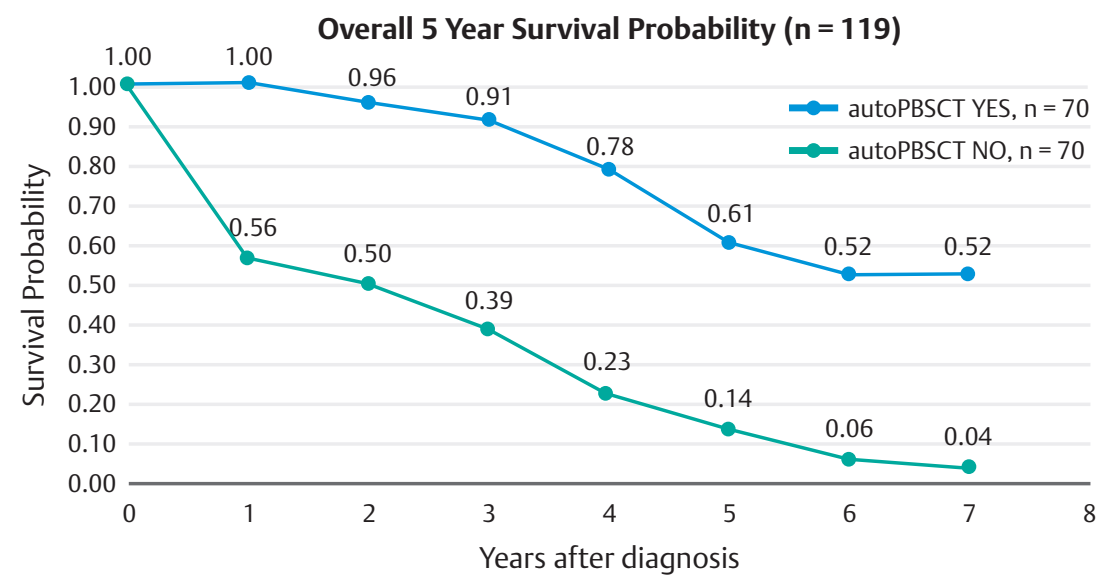

> Fig. 4 5-year probability survival rate for Ewing sarcoma patients, autoPBSCT $(n=23)$ vs. non-autoPBSCT ( $=96), 1990-2017$.
The 5-year probability overall survival rate for Ewing sarcoma family of tumors $(n=119)$ is $49 \%$, number of survivors is 54 ( $\triangleright$ Fig. 3 ). $54 \%(n=28)$ of females have survived and $38 \%(n=26)$ of males. Children younger than 10 years had $64 \%(n=29)$ survival rate in contrast to older children with $33 \%$ $(n=25)$. The survival rate for localised disease was $71 \%(n=27), 33 \%(n=27)$ for locally disseminated and $24 \%(n=7)$ for the group of patients who developed metastases during the course of the disease. The survival for patients ( $\triangleright$ Table 2 ) with good chemotherapy response is $75 \%(n=41)$ and 8,6\% $(n=2)$. 5-year probability survival rate for patients who proceed with the auto PBSCT was $52 \%(n=23)$ in contrast to patients who didn't 4\% ( $n=96$; > Fig. 4 ).

\section{Discussion}

For the period of last 29 years after the multimodal approach to treatment of osteosarcoma have been established, there was also several new studies which have found the implementation in treatment. So, the overall survival rate of $60 \%$ could be explained with changing the protocol scheme in treatment, different drugs implementation. Rosen $T$ and COSS scheme protocol was implemented from 1990-2004, and from 2005 the EURAMOS was the treatment study.

Analyzing the risk factors at the time of diagnosis the best survival of $82 \%$ had patients with localized disease what was expected, but also rather high incidence of patients with locally disseminated disease $(n=70)$ of 140 patients.

In order to analyse response to chemotherapy $84 \%$ of patients with good response (HUVOS grade III and IV) have survived, and only $32 \%$ with poor grade response (HUVOS grade I and II). Which was also expected and according to literature.

Regarding the Ewing sarcoma family the overall 5-year survival probability rate is $49 \%$ ( Fig. 3), but also the Euro EWING 99 scheme was implemented in 2000 , and in 2009 the EWING 2008 - the results are seen in 5-year survival probability rate in comparison of patients who had high dose chemotherapy and blood autologous stem cell rescue with standard chemotherapy ( $>$ Fig.4), 
- Tab.2 Characteristics of Ewing sarcoma patients, 1990-2017.

\begin{tabular}{|c|c|c|}
\hline Demographic and diagnostic data & $\begin{array}{l}\text { No of patients } \\
(n=119)\end{array}$ & $\begin{array}{l}\text { No of survivors } \\
(n=54)\end{array}$ \\
\hline \multicolumn{3}{|l|}{ 1. Sex } \\
\hline Male & 68 & $38 \%(n=26)$ \\
\hline Female & 51 & $54 \%(n=28)$ \\
\hline \multicolumn{3}{|l|}{ 2. Age at diagnosis } \\
\hline$\leq 10 \mathrm{y}$ & 45 & $64 \%(n=29)$ \\
\hline$>10 y$ & 74 & $33 \%(n=25)$ \\
\hline \multicolumn{3}{|l|}{ 3. Disease } \\
\hline localised (at time of diagnosis) & 38 & $71 \%(n=27)$ \\
\hline local. disseminated (at time of diagnosis) & 81 & $33 \%(n=27)$ \\
\hline metastatic (during course of disease) & 29 & $24 \%(n=7)$ \\
\hline 4. Response to Chemo HUVOS & $\mathrm{n}=64$ & $51,5 \%(n=33)$ \\
\hline poor (grade I+II) & 23 & $8,6 \%(n=2)$ \\
\hline good (grade III + IV) & 41 & $75 \%(n=31)$ \\
\hline *no data & $\mathrm{n}=55$ & \\
\hline
\end{tabular}

*due to metastatic disease/local invasion -mutilation hazard/primary resection

survival rate for autoPBSCT patients is $52 \%$ in contrast to those who had standard treatment [3]. The patients who had high-dose chemotherapy and blood autologous stem cell rescue were in complete or partial remission before the procedure, and had tumor volume $>400 \mathrm{ml}$ and/or metastatic disease at diagnosis.

Smaller number of analysed Ewing sarcoma family of tumors due to response to chemotherapy can be explained by metastatic or inoperable disease, but most of all with primary tumor extirpation which was done before the patient's admission to our hospital.

\section{Conclusion}

Due to multimodal therapy application in treatment of rare bone tumors in childhood over the years, we have evidence of upgrading the treatment results, but this result will be much more improved in more active participation in the international bone tumor studies.

\section{Authors}

\section{Bonevski A}

Children's Hospital Zagreb, Oncology

department, Zagreb, Croatia

\section{Stepan Giljević J}

Children's Hospital Zagreb, Oncology

department, Zagreb, Croatia

\section{Ćepulić M}

Children's Hospital Zagreb,

Oncology department, Zagreb, Croatia

\section{Seiwerth S}

Institute of Pathology School of Medicine University of Zagreb, Zagreb, Croatia

\section{Corresponding Author}

Dr. med. Aleksandra Bonevski

Ul. Vjekoslava Klaića 16

10000 Zagreb

Croatia

\section{References}

[1] Mirabello L, Troisi RJ, Savage SA. Osteosarcoma Incidence and Survival Rates From 1973-2004: data from SEER. Cancer 2009; 1531-1543

[2] Esiashvili N, Goodman M, Marcus RB Jr. Changes in incidence and survival of Ewing sarcoma patients over the past 3 decades: SEER data. J Pediatr Hematol Oncol 2008; 30 (6): 425-430

[3] Bernstein M, Kovar H, Paulussen M et al. Ewing sarcoma family of tumors: Ewing sarcoma of bone and soft tissue and the peripheral primitive neuroectodermal tumors. In: Pizzo P, Poplack D, eds. Principles and Practice of Pediatric Oncology. $5^{\text {th }}$ ed. Philadelphia, PA: Lippincott Wiliams and Wilkins; 2005: 1002-1032
IMPRESSUM

Verantwortlich für den Inhalt Prof. Dr. Daniel Baumhoer, Basel Prof. Dr. Gernot Jundt, Basel 